\title{
Potential tumor suppressing role of microRNA-545 in epithelial ovarian cancer
}

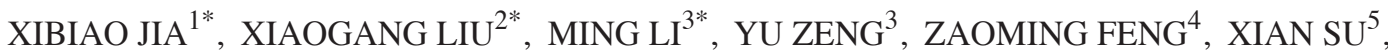 \\ YAN HUANG $^{6}$, MAOMAO CHEN $^{3}$ and XUEYI YANG ${ }^{7}$ \\ ${ }^{1}$ Key Laboratory of Birth Defects and Related Diseases of Women and Children, Ministry of Education, \\ West China Second University Hospital, Sichuan University, Chengdu, Sichuan 610041; \\ ${ }^{2}$ Department of Obstetrics and Gynaecology, People's Hospital of Yuxi, Yuxi, Yunnan 653100; \\ ${ }^{3}$ Centre for Disease Prevention and Control, Chengdu Military Region; ${ }^{4}$ Department of Gastroenterology, \\ The People's Liberation Army 452 Hospital; ${ }^{5}$ Department of Internal Medicine, The Third People's Hospital, Chengdu, \\ Sichuan 610021; ${ }^{6}$ Department of Neurology, The Third People's Hospital, Chengdu, Sichuan 610031; \\ ${ }^{7}$ Department of Inspection and Pharmacy, Jiangsu College of Nursing, Huaian, Jiangsu 223001, P.R. China
}

Received May 25, 2016; Accepted January 5, 2018

DOI: $10.3892 / \mathrm{ol} .2018 .8130$

\begin{abstract}
Epithelial ovarian cancer (EOC) is the most common type of ovarian cancer, which exhibits invasive traits. MicroRNAs (miRNAs/miRs) have been demonstrated to serve important functions in the pathogenesis of EOC. However, the function of miR-545 in EOC remains unknown. In the present study, the function of miR-545 in EOC was analyzed and it was identified that miR-545 is downregulated in EOC tissues and cell lines. Additionally, a low level of miR-545 expression was associated with a low survival rate of patients with EOC. Furthermore, overexpression of miR-545 inhibited cell growth and promoted apoptosis. Suppression of miR-545 promoted cell growth and inhibited apoptosis. Additionally, the RAC- $\gamma$ serine/threonine-protein kinase gene was targeted by miR-545. Thus, it may be concluded that miR-545 exhibited antitumor traits in EOC.
\end{abstract}

Correspondence to: $\mathrm{Dr}$ Maomao Chen, Centre for Disease Prevention and Control, Chengdu Military Region, 12 Tianxian North Road, Chengdu, Sichuan 610021, P.R. China

E-mail: chenmaomaocdc@126.com

Dr Xueyi Yang, Department of Inspection and Pharmacy, Jiangsu College of Nursing, 9 Avenue of Science and Technology, Huaian, Jiangsu 223001, P.R. China

E-mail: yangxueyi@163.com

*Contributed equally

Key words: microRNA-545, epithelial ovarian cancer, tumor suppressor

\section{Introduction}

Epithelial ovarian cancer (EOC) is the most common type of invasive ovarian cancer, and a leading cause of cancer-associated mortalities in females globally $(1,2)$. In China, the incidence rate of ovarian cancer was 7.91/100,000 and the age-adjusted incidence rate was 5.35/100,000 between 1999 and 2010 (3). The incidence of EOC increases rapidly following menopause (4). Although a number of factors are involved the in the molecular mechanism behind EOC, these risk factors are not well defined and the pathogenesis of EOC remains unclear (5). The progress in the treatment of EOC is not optimistic, the prognosis is poor and the 5-year relative survival rate is $\sim 30 \%$ (6).

In recent years the functions of microRNAs (miRNAs/miRs) in the cancer have begun to be revealed (7-12), and a number of miRNAs have been identified to be involved in the pathogenesis of EOC (13-19). miRNAs are evolutionarily conserved, small non-coding RNAs that regulate the expression of genes and translocation of proteins (20).

The functions of miR-545 have been studied in several types of cancer. In pancreatic ductal adenocarcinoma, miR-545 inhibited cellular proliferation and was associated with a decreased survival rate in patients (21). In lung cancer, miR-545 suppressed cellular proliferation via the inhibition of cyclin D1 and cyclin-dependent kinase 4 expression (22). However, the function of miR-545 in EOC remains unknown.

In the present study, the expression level of miR-545 was analyzed in EOC tissues and the in vitro functions of miR-545 were investigated. Subsequently, the potential miR-545-target genes were assessed.

\section{Materials and methods}

Patients and tissues. A total of 27 EOC tissues and matched normal adjacent tissues were collected from 27 subjects (the mean age was 59.9 years, and the range was $36-45$ years) who underwent surgery for the treatment of EOC at the West 
China Women's and Children's Hospital of Sichuan University (Chengdu, China). The histopathological diagnosis of EOC was provided and confirmed by the senior pathologist of the West China Second University Hospital, Sichuan University (Chengdu, Sichuan 610041). The survival status of patients was recorded and used for Kaplan-Meier survival analysis. Patient information is summarized in Table I. The present study was approved by Ethic Committee of Sichuan University and each patient provided written informed consent.

Cell lines. The EOC SKOV3 and HO8910 cell lines and the normal immortalized human ovarian surface epithelial cells IOSE29 were acquired from the Type Culture Collection of the Chinese Academy of Sciences (Shanghai, China). Cells were cultured in RPMI-1640 medium with $10 \%$ fetal bovine serum (Invitrogen; Thermo Fisher Scientific, Inc., Waltham, MA, USA) (23).

Reverse transcription-quantitative polymerase chain reaction $(R T-q P C R)$. Following cell and tissue isolation, total RNA was purified from EOC tissues, IOSE29, SKOV3, HO8910 cell lines (treated or control group) using TRIzol reagent (Life Sciences; Thermo Fisher Scientific, Inc.) according to the manufacturer's protocol. The extracted RNA was dissolved in DEPC-treated $\mathrm{ddH}_{2} \mathrm{O}$ and subjected to DNAse I treatment (catalog no, EN0521; Invitrogen; Thermo Fisher Scientific, Inc.). Total RNA was reverse-transcribed to cDNA by using All-in-One ${ }^{\mathrm{TM}}$ miRNA First-Strand cDNA Synthesis kit (catalog no. AMRT-0020; Biocompare, South San Francisco, CA, USA). The PCR process was completed by SYBR ${ }^{\circledR}$ Green PCR Master Mix (catalog no. 4364344; Thermo Fisher Scientific, Inc.). The primers were synthesized and tested by the Chengdu Haoyi Biotechnology Co., Ltd (http://haoyibio.net) (Chengdu, China). The following primers were used for the analysis: miR-545, 5S rRNA forward primer, 5'-ACGGCCATACCACCCTGAAC-3', reverse primer, 5'-GGCGGTCTC CCATCCAAGTA-3'; U6 forward primer, 5'-CTCGCTTCGGCAGCACA-3', reverse primer, 5'-AACGCT TCACGAATTTGCGT-3'. Data analysis were performed using the the $2^{-\triangle \Delta \mathrm{Cq}}$ method of normalization (24). U6 was used for the normalization of miR-545 (10,25-27).

miR-545 mimics and anti-sense oligonucleotides transfection (ASO). The miR-545 mimics (5'-UCAGCAAACAUUUAU UGUGUGC-3'), miR-545 ASO (5'-GCACACAAUAAAUGU UUGCUGA-3') and negative controls (5'-UGGGCGUAU AGACGUGUUACAC-3') were purchased from Dharmacon (GE Healthcare Life Sciences, Little Chalfont, UK). Cells were seeded at $2 \times 10^{5}$ per well in 6-well plates for further investigation. These cells were transfected with miR-545 mimics, miR-545 ASO or miR-545 mimics using Lipofectamine 2000 (Invitrogen; Thermo Fisher Scientific, Inc.) according to the manufacturer's protocol. After $24 \mathrm{~h}$, the transfected cells were used for further experiments.

Cell proliferation assay. The cellular proliferation was assessed using an MTT assay. SKOV3 and HO8910 cells were seeded into 96-well plates at a density of $5 \times 10^{5}$ cells/well. MTT reagent was added into the medium at a final concentration of $0.1 \mathrm{mg} / \mathrm{ml}$ and the plate was incubated at $37^{\circ} \mathrm{C}$ for $3 \mathrm{~h}$. Following incubation, Dimethyl sulfoxide $(100 \mu \mathrm{l})$ was added at $37^{\circ} \mathrm{C}$ for $15 \mathrm{~min}$.
Optical density was measured using a microplate reader at $570 \mathrm{~nm}$.

Cell apoptosis analysis. Annexin V-fluorescein isothiocyanate (FITC) Staining/Detection kit (ab14085, Abcam, Cambridge, UK) was used. The binding buffer were prepared according to the manufacturer's protocol. Treated SKOV3 and HO8910 cells were suspended $\left(5 \times 10^{5}\right.$ cells $\left./ \mathrm{ml}\right)$ in 1 xbinding buffer. Annexin V-FITC (5 $\mu \mathrm{l})$ was added and incubated for $15 \mathrm{~min}$ at room temperature. Subsequently, propidium iodide (5 $\mu \mathrm{l})$ (ab14083, Abcam) was added for $5 \mathrm{~min}$ at room temperature, samples were analyzed with a flow cytometer using the $488 \mathrm{~nm}$ excitation line (Argon-ion laser or solid state laser) and emission detected at $530 \mathrm{~nm}$ (green, FITC) and 575-610 nm (orange, PI). The data was analyzed by BD LSR II software (v.1.1.0, Franklin, NJ, USA).

Dual luciferase reporter assays. SKOV3 cells were seeded at $1 \times 10^{5}$ per well and were serum-starved for $6 \mathrm{~h}$ prior to transfection. The RAC- $\gamma$ serine/threonine-protein kinase (AKT3) 3'-UTR reporter plasmid (500 ng) and the pGL3-control (100 ng) (Promega Corporation, Madison, WI, USA) were co-transfected into cells using Lipofectamine 2000 (Invitrogen; Thermo Fisher Scientific, Inc.). Cells were harvested and luciferase activities were analyzed after $24 \mathrm{~h}$ using the Dual-Luciferase Reporter Assay system (Promega Corporation). Mutants of AKT3 3'-UTR were generated using a Site-Directed Mutagenesis kit (Promega Corporation). The data was normalized by Renilla luciferase activity. The value in miR-NC group was arbitrarily defined as $100 \%$.

Prediction of the possible targets of $m i R-545$. Targetscan software (http://www.targetscan.org/) was used to predict the possible targets of miR-545 by searching miR-545. TargetScan is online software, that predicts biological targets of miRNA by searching for the presence of conserved $8 \mathrm{mer}, 7 \mathrm{mer}$, and $6 \mathrm{mer}$ sites that match the seed region of each miRNA (28).

Western blot analysis. The lysates were prepared with lysis buffer (M-PER ${ }^{\mathrm{TM}}$ Mammalian Protein Extraction Reagent, Thermo Fisher Scientific, Inc., Waltham, MA, USA) containing protease inhibitors (Pierce Protease Inhibitor, A32963, Thermo Fisher Scientific, Inc.) and then were cleared by centrifugation. The protein concentration was assayed by Pierce ${ }^{\mathrm{TM}}$ Rapid Gold BCA Protein Assay Kit (Thermo Fisher Scientific, Inc.). Total proteins $(30 \mu \mathrm{g})$ were resolved using SDS-PAGE (10\%) and transferred onto polyvinylidene difluoride membranes (Merck KGaA, Darmstadt, Germany). The membranes were immersed with 5\% non-fat dry milk (in Blocking Buffer, Thermo Fisher Scientific,Inc.) for $15 \mathrm{~min}$ at room temperature. Membranes were washed with PBS for $15 \mathrm{~min}$ and then incubated with primary antibodies overnight in $4^{\circ} \mathrm{C}$, washed by PBS for three times. Then the membranes were incubated with secondary antibodies for $2 \mathrm{~h}$ at room temperature. Western blot Signal was developed by Pierce ${ }^{\mathrm{TM}}$ DAB Substrate Kit (Thermo Fisher, Scientific, Inc.), then the blotting images were captured using CL-XPosure ${ }^{\mathrm{TM}}$ film 5x7 inch (Thermo Fisher Scientific, Inc.). The following antibodies were used as follows: AKT3 (catalog no. sc-56878, 1:1,000 dilution; Santa Cruz Biotechnology, Inc., Dallas, TX, USA), GAPDH (catalog no. sc69778, 1:3,000 dilution; Santa 
Table I. Characteristics of patients with ovarian cancer.

\begin{tabular}{rclccc}
\hline No. & Age, years & Type & Stage & Follow-up time, months & Status \\
\hline 1 & 45 & Epithelial ovarian cancer/serous & IV & 34 & Deceased \\
2 & 54 & Epithelial ovarian cancer/serous & IV & 44 & Deceased \\
3 & 46 & Epithelial ovarian cancer/serous & III & 55 & Deceased \\
4 & 56 & Epithelial ovarian cancer/serous & III & 66 & Survived \\
5 & 48 & Epithelial ovarian cancer/serous & IV & 66 & Survived \\
6 & 36 & Epithelial ovarian cancer/serous & III & 66 & Survived \\
7 & 57 & Epithelial ovarian cancer/serous & III & 66 & Survived \\
8 & 45 & Epithelial ovarian cancer/serous & IV & 66 & Survived \\
9 & 64 & Epithelial ovarian cancer/serous & IV & 66 & Survived \\
10 & 75 & Epithelial ovarian cancer/serous & IV & 66 & Survived \\
11 & 56 & Epithelial ovarian cancer/serous & IV & 66 & Survived \\
12 & 74 & Epithelial ovarian cancer/serous & IV & 66 & Survived \\
13 & 69 & Epithelial ovarian cancer/serous & IV & 66 & Survived \\
14 & 79 & Epithelial ovarian cancer/serous & IV & 4 & Deceased \\
15 & 75 & Epithelial ovarian cancer/serous & IV & 7 & Deceased \\
16 & 67 & Epithelial ovarian cancer/serous & IV & 11 & Deceased \\
17 & 65 & Epithelial ovarian cancer/serous & III & 13 & Deceased \\
18 & 69 & Epithelial ovarian cancer/serous & IV & 14 & Deceased \\
19 & 73 & Epithelial ovarian cancer/serous & IV & 19 & Deceased \\
20 & 65 & Epithelial ovarian cancer/serous & IV & 22 & Deceased \\
21 & 59 & Epithelial ovarian cancer/serous & IV & 27 & Deceased \\
22 & 57 & Epithelial ovarian cancer/serous & III & 66 & Deceased \\
23 & 63 & Epithelial ovarian cancer/serous & IV & 66 & Survived \\
24 & 67 & Epithelial ovarian cancer/serous & IV & 66 & Survived \\
25 & 54 & Epithelial ovarian cancer/serous & III & 66 & Survived \\
26 & 51 & Epithelial ovarian cancer/serous & IV & 66 & Survived \\
27 & 49 & Epithelial ovarian cancer/serous & IV & 66 & Survived \\
\hline
\end{tabular}

Cruz Biotechnology, Inc.), goat anti-rabbit (catalog no. sc-2004, 1:10,000 dilution; Santa Cruz Biotechnology, Inc.).

Statistical analysis. Data are presented as mean \pm standard deviation. Statistical analysis was performed using SPSS software (version 10; SPSS, Inc., Chicago, IL, USA). The difference between two groups was analyzed using a two-tailed Student's t-test. One- way analysis of variance (ANOVA) was used to analyze the difference between three groups; Fisher's least significant difference test was performed following ANOVA. Analysis of the survival rate of patients with EOC was performed via Kaplan-Meier survival curves and a log-rank test was performed to confirm the significance. $\mathrm{P}<0.05$ was considered to indicate a statistically significant difference.

\section{Results}

miR-545 expression is decreased in EOC tissues and low level of miR-545 is associated with low survival rate of patients with $E O C$. The miR-545 levels in 27 EOC tissues samples were analyzed using RT-qPCR. The levels of miR-545 expression were decreased in tumor tissues compared with matched-adjacent normal tissues in 23 specimens (Fig. 1A). The mean expression of miR-545 in the 27 EOC tissues samples was significantly lower than that in the adjacent normal samples (Fig. 1B). The survival rate of the patients for 66 months following surgical treatment was also assessed, and the 27 patients with EOC were divided into two groups according to the median value of the miR-545 expression in EOC tissues. Kaplan-Meyer survival analysis revealed that patients expressing increased levels of miR-545 in EOC tissues were associated with increased survival rates (Fig. 1C).

Overexpression of miR-545 inhibits cells growth and promotes apoptosis. Next the function of miR-545 in the EOC cell lines SKOV3 and HO8910 was tested. It was identified that miR-545 was expressed at decreased levels in SKOV3 and HO8910 cells compared with normal ovarian tissue and the normal immortalized human ovarian surface epithelial cells IOSE29 (Fig. 2A). Next the miR-545 levels in SKOV3 and HO8910 cells were determined following transfection with miR-545 mimics; miR-545 mimics upregulated the miR-545 levels in the two cell lines at 48 and $72 \mathrm{~h}$ after transfection (Fig. 2B). Cellular proliferation and apoptosis were assessed using MTT analysis at $48 \mathrm{~h}$ after transfection with miR-545 mimics or the negative control. Overexpression of miR-545 was revealed to inhibit cellular proliferation (Fig. 2C). At $48 \mathrm{~h}$ after transfection, the rates of cell apoptosis were assessed using flow cytometry, and it was 


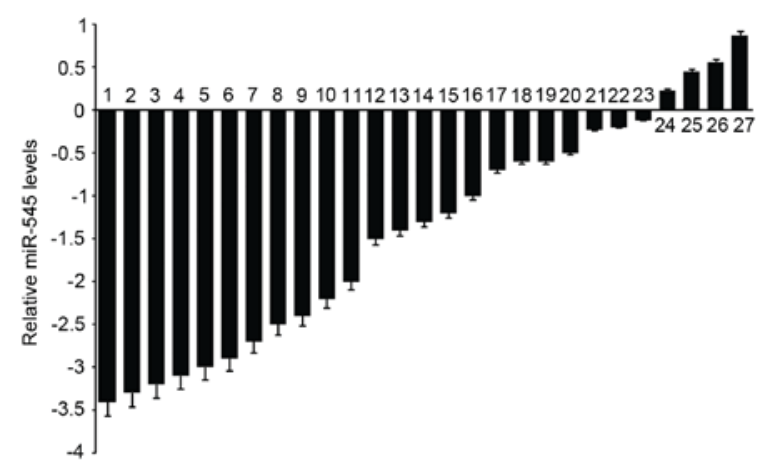

C

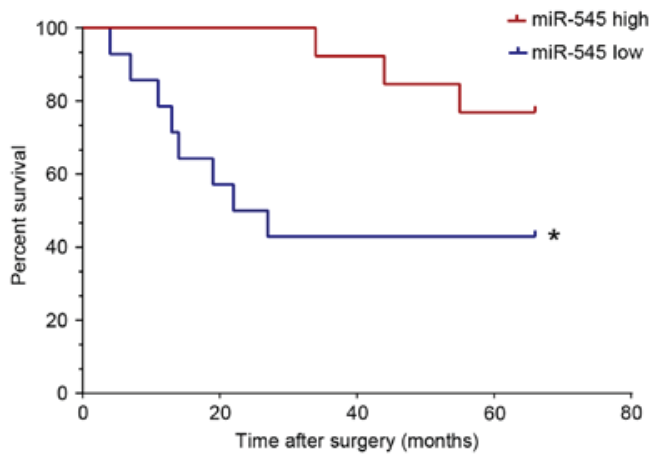

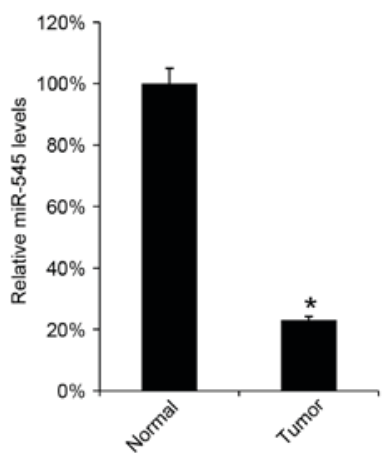

Figure 1. Low levels of miR-545 in EOC tissues are associated with low survival rates of patients with EOC. (A) Reverse transcription-quantitative polymerase chain reaction experiments were performed to assess the expression of miR-545 in EOC tissues. U6 small nuclear RNA was used as blank control. The values are relative to the expression of miR-545 [ $\log 2$ (Tumor/Normal)]. (B) The mean value of miR-545 levels in EOC tissues and the adjacent normal tissues were calculated. (C) The median values of miR-545 expression in 27 EOC tissues was used as the cutoff point separating miR-545-high-expression group (n=13) from the miR-545-low-expression group $(n=13)$. A Kaplan-Meier survival curve was produced. The data are presented as the mean \pm standard deviation. The same experiment was repeated at least three times. ${ }^{*} \mathrm{P}<0.05$. miR-545, microRNA-545; EOC, epithelial ovarian cancer.

A
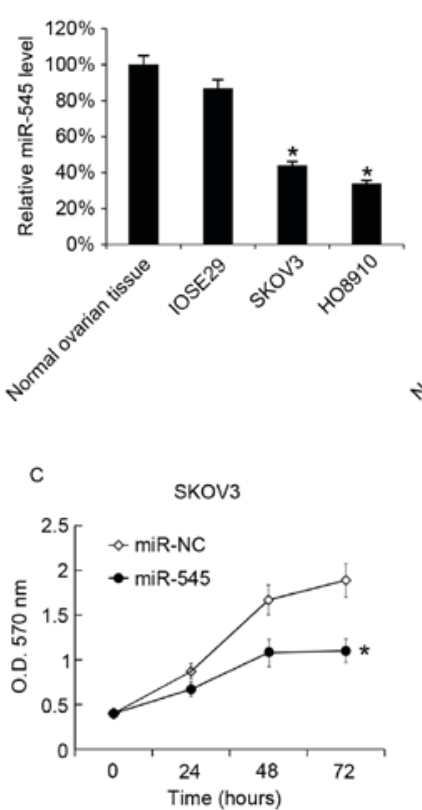

B
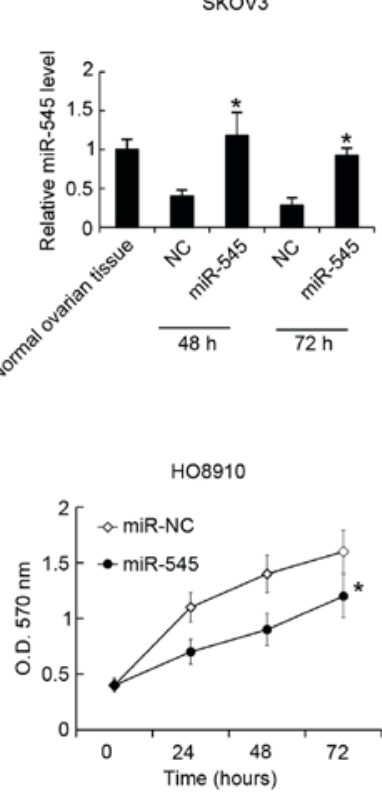

HO8910
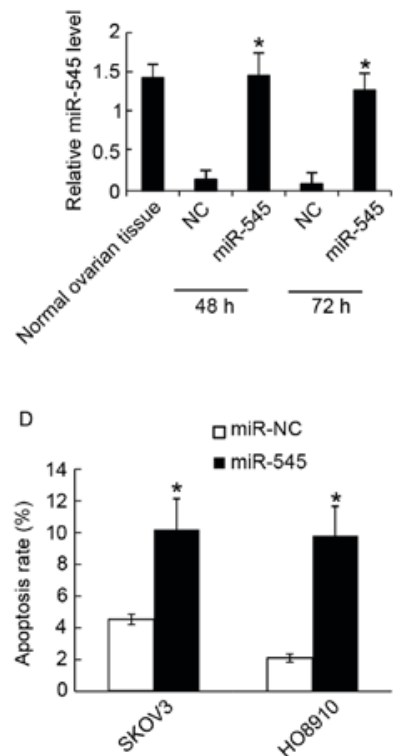

Figure 2. Transfection of miR-545 mimics inhibits cell growth and promotes apoptosis. (A) The miR-545 levels in normal ovarian tissues; IOSE29, SKOV3 and HO8910 cells were analyzed using RT-qPCR. The miR-545 level in normal ovarian tissues was arbitrarily defined as $100 \%$. (B) miR-545 mimics were transfected into SKOV3 and HO8910, and after 48 and $72 \mathrm{~h}$ the miR-545 levels were analyzed using RT-qPCR. Levels of miR-545 expression in normal ovarian tissues was arbitrarily defined as $100 \%$. (C) Following transfection with miR-NC or miR-545 mimic, cellular proliferation was analyzed using an MTT assay at 24, 48 and 72 h, (D) At $48 \mathrm{~h}$ after transfection with the miR-545 mimic, cell apoptosis was measured using annexin V-propidium iodide staining. Data are presented as the mean \pm standard deviation. The experiment was repeated at least three times. ${ }^{*} \mathrm{P}<0.05$ vs. NC. RT-qPCR, reverse transcription-quantitative polymerase chain reaction; miR-545, microRNA-545; OD, optical density; NC, negative control. 
A

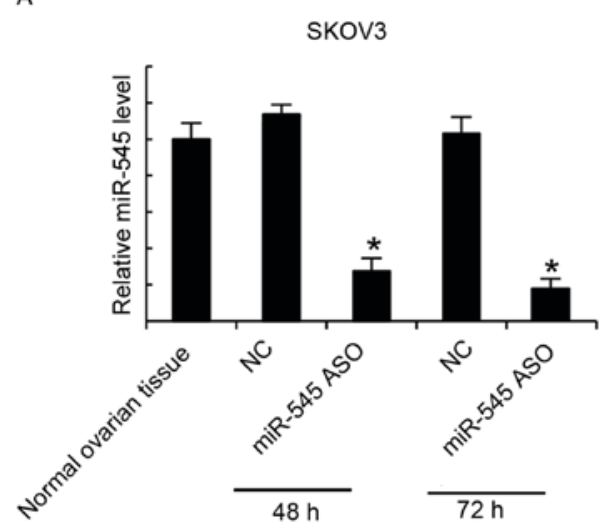

B

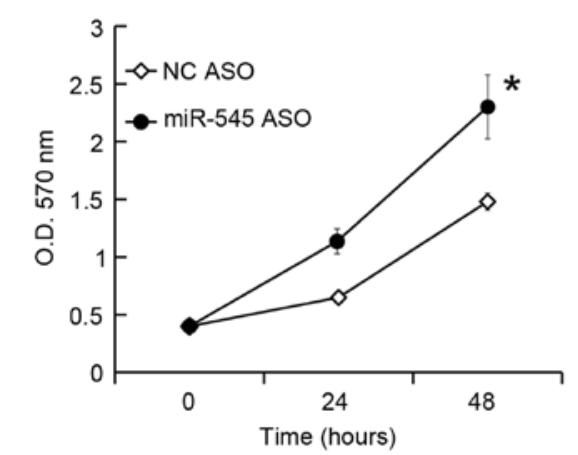

C

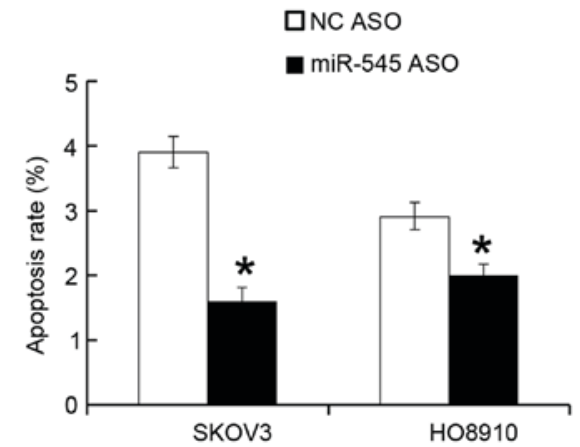

HO8910

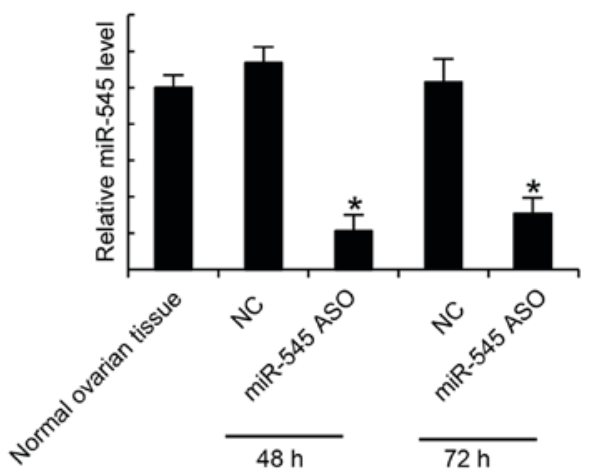

HO8910

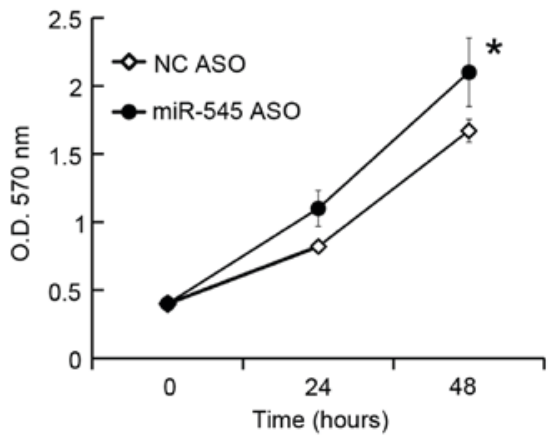

Figure 3. Transfection of miR-545 ASO promotes cell growth and inhibits apoptosis. (A) miR-545 ASO were transfected into SKOV3 and HO8910 cells, and 48 and $72 \mathrm{~h}$ later, the miR-545 levels were analyzed by reverse transcription-quantitative polymerase chain reaction. The miR-545 level in normal ovarian tissues was arbitrarily defined as $100 \%$. (B) At 24,48 and $72 \mathrm{~h}$ after transfection with miR-NC ASO or miR-545 mimic ASO, the cellular proliferation was analyzed using an MTT assay. (C) The cell apoptosis was measured using annexin V-propidium iodide staining $48 \mathrm{~h}$ after miR-545 ASO transfection. The data are the mean \pm standard deviation. The experiment was repeated at least three times. "P<0.05 vs. NC. miR-545, microRNA-545; anti-sense oligonucleotides ASO; OD, optical density; NC, negative control.

identified that miR-545 increased the apoptosis rate in SKOV3 and HO8910 (Fig. 2D).

Suppression of miR-545 promoted cell proliferation and inhibited apoptosis. The miR-545 levels in SKOV3 and HO8910 were suppressed by miR-545 ASO transfection. After 48 and $72 \mathrm{~h}$, the miR-545 levels were assayed using RT-qPCR. It was identified that miR-545 ASO significantly suppressed the miR-545 levels at the two time points (Fig. 3A). The MTT assay revealed that miR-545 ASO transfection promoted cellular proliferation in SKOV3 and HO8910 cells (Fig. 3B). Similarly, $48 \mathrm{~h}$ after transfection, the cell apoptosis rates were assessed using FACS analysis, and it was revealed that miR-545 ASO decreased the apoptosis rate in SKOV3 and HO8910 cells (Fig. 3C).

AKT3 is targeted by miR-545. The phosphatidylinositol 3-kinase (PI3K)/AKT pathway has been demonstrated to serve a function in oncogenic transformation in ovarian cancer, with AKT3 
A

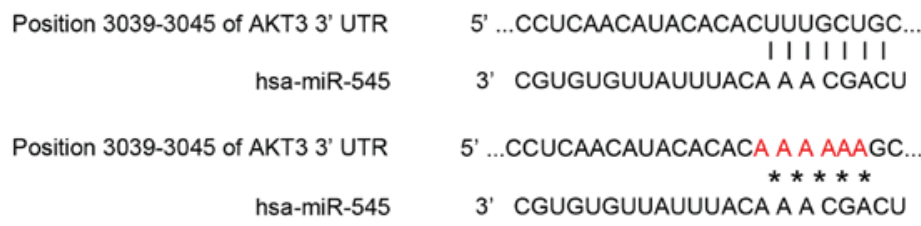

B

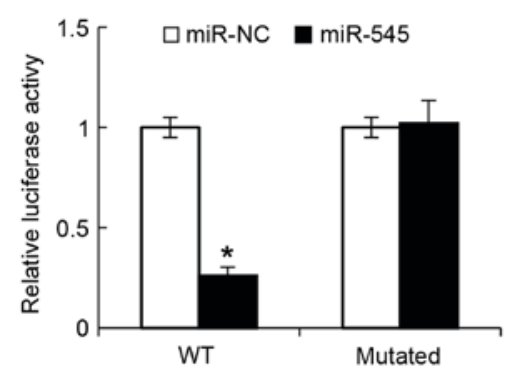

C

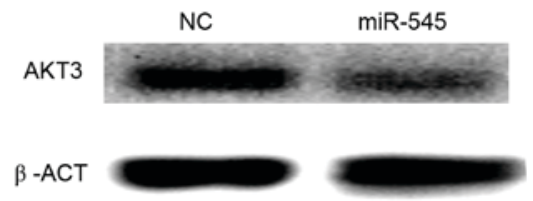

Figure 4. AKT3 is targeted by miR-545. (A) The binding site and its mutated sequence are presented, and the mutated sites are annotated. (B) miR-545 inhibited the activity of luciferase containing the 3'-UTR of the AKT3 gene and did not inhibit the mutated version. (C) SKOV3 cells were transfected with miR-545 mimics and $48 \mathrm{~h}$ later, the abundance of AKT3 protein was assessed using western blotting. The experiment was repeated at least three times. ${ }^{*}<0.05$. miR-545, microRNA-545; WT, wild type; AKT3, RAC- $\gamma$ serine/threonine-protein kinase; 3'-UTR, 3'-untranslated region; NC, negative control.

being an important mediator of ovarian oncogenesis (29). The bioinformatics algorithm suggested that miR-545 may bind the 3'-UTR of AKT3 (Fig. 4A). The intact 3'-UTR of AKT3 and its mutated version were cloned into luciferase reporter plasmids and used for co-transfection with miR-545 into IOSE29 cells. It was revealed that miR-545 decreased the luciferase activity of the wild type 3'-UTR reporter, whereas the luciferase activity of the reporter with the mutated version was not significantly different between miR-NC and miR-545 (Fig. 4B). At $48 \mathrm{~h}$ after transfection of the miR-545 mimics into the SKOV3 cells, AKT3 protein levels in the SKOV3 cells were assayed by western blot analysis, and found that these data indicated that miR-545 exerted its function through AKT3. Transfected miR-545 mimics decreased the AKT3 protein levels (Fig. 4C).

\section{Discussion}

miRNAs have been demonstrated to be involved in the pathogenesis of EOC (30-32). A previous study by the authors revealed the function of miR-494 in EOC (33). The authors demonstrated that miR-494 was expressed at low levels in EOC tissues and cells. Overexpression of miR-494 inhibited the proliferation and migration of the EOC cells. miR-494 was revealed to target Myc proto-oncogene protein (34). In the present study, the function of miR-545 in EOC was analyzed and it was demonstrated that miR-545 served an antitumor function, and AKT3 was targeted by miR-545.

miR-545 appears to function as a cancer suppressor gene in a number of types of cancer, including pancreatic ductal adenocarcinoma and lung cancer $(22,35)$. In a previous study, inhibition of miR-545 expression decreased the radiosensitivity of Lewis lung carcinoma, with miR-545 regulating Ku70 expression by targeting the 3'-UTR of $\mathrm{Ku} 70$, a process that was involved in radiotherapy (36). On the one hand, miR-545 can inhibit the proliferation of cancer cells by targeting oncogenes (35); however, miR-545 could also enhance radiosensitivity during cancer therapy. Thus, miR-545 is a good candidate target for cancer therapy.

Expression of AKT3 is increased in several types of cancer (37). In triple negative breast cancer (TNBC), the downregulation of AKT3 inhibits the cellular proliferation of TNBC cell lines and depletion of AKT3 in TNBC sensitizes cells to the pan-AKT inhibitor GSK690693 (38). Additionally, in prostate cancer, AKT3 promotes the proliferation of prostate cancer proliferation cells through regulation of $\mathrm{AKT}$, serine/threonine protein kinase B-Raf and tuberous sclerosis 1/2 (39).

In conclusion, the data collected in the present study demonstrated that miR-545 may serve an antitumor function in EOC.

\section{Acknowledgements}

The authors would like to thank Ms Gao Hui (Key Laboratory of Birth Defects and Related Diseases of Women and Children, Ministry of Education, West China Second University Hospital, Sichuan University, Chengdu, China) for her technical assistance.

\section{Funding}

No funding was received.

\section{Availability of data and materials}

All data generated or analyzed during this study are included in this published article.

\section{Author's contributions}

XJ collected patient data and performed cell experiments. XL analyzed the data. ML performed the statistical analysis. YZ performed the literature search and analyzed the data. ZF 
performed cell experiments. XS performed flow cytometry. YH collected and analyzed the data. MC contributed to study design and submitted the manuscript. XY contributed to study design and manuscript writing.

\section{Ethics approval and consent to participate}

The present study was approved by Ethic Committee of Sichuan University and each patient provided written informed consent.

\section{Consent for publication}

All samples were collected with the informed consent of the patients.

\section{Competing interests}

The authors declare that they have no competing interests.

\section{Reference}

1. Copeland LJ: Epithelial ovarian cancer. Clin Gynecol Oncol 7: 313-367, 2007.

2. Holschneider $\mathrm{CH}$ and Berek JS: Ovarian cancer: Epidemiology, biology, and prognostic factors. Semin Surg Oncol 19: 3-10, 2000

3. Wang B, Liu SZ, Zheng RS, Zhang F, Chen WQ and Sun XB: Time trends of ovarian cancer incidence in China. Asian Pac J Cancer Prev 15: 191-193, 2014.

4. Shushan A, Paltiel O, Iscovich J, Elchalal U, Peretz T and Schenker JG: Human menopausal gonadotropin and the risk of epithelial ovarian cancer. Fertil Steril 65: 13-18, 1996.

5. Daniilidis A and Karagiannis V: Epithelial ovarian cancer. Risk factors, screening and the role of prophylactic oophorectomy Hippokratia 11: 63-66, 2007.

6. Survival of Cancer Patients in Europe: The EUROCARE-2 study. IARC Sci Publ 1-572, 1999.

7. Garzon R, Calin GA and Croce CM: MicroRNAs in Cancer. Annu Rev Med 60: 167-179, 2009.

8. Ambros V: The functions of animal microRNAs. Nature 431: 350-355, 2004.

9. Hou J, Lin L, Zhou W, Wang Z, Ding G, Dong Q, Qin L, Wu X, Zheng Y, Yang Y, et al: Identification of miRNomes in human liver and hepatocellular carcinoma reveals miR-199a/b-3p as therapeutic target for hepatocellular carcinoma. Cancer Cell 19: 232-243, 2011.

10. Li D, Liu X, Lin L, Hou J, Li N, Wang C, Wang P, Zhang Q, Zhang P, Zhou W, et al: MicroRNA-99a inhibits hepatocellular carcinoma growth and correlates with prognosis of patients with hepatocellular carcinoma. J Biol Chem 286: 36677-36685, 2011.

11. Mendell JT and Olson EN: MicroRNAs in stress signaling and human disease. Cell 148: 1172-1187, 2012.

12. Croce CM: Causes and consequences of microRNA dysregulation in cancer. Nat Rev Genet 10: 704-714, 2009.

13. Iorio MV, Visone R, Di Leva G, Donati V, Petrocca F, Casalini P, Taccioli C, Volinia S, Liu CG, Alder H, et al: MicroRNA signatures in human ovarian cancer. Cancer Res 67: 8699-8707, 2007

14. Resnick KE, Alder H, Hagan JP, Richardson DL, Croce CM and Cohn DE: The detection of differentially expressed microRNAs from the serum of ovarian cancer patients using a novel real-time PCR platform. Gynecol Oncol 112: 55-59, 2009.

15. Chung YW, Bae HS, Song JY, Lee JK, Lee NW, Kim T and Lee KW: Detection of microRNA as novel biomarkers of epithelial ovarian cancer from the serum of ovarian cancer patients. Int J Gynecol Cancer 23: 673-679, 2013.

16. Shapira I, Oswald M, Lovecchio J, Khalili H, Menzin A, Whyte J, Dos Santos L, Liang S, Bhuiya T, Keogh M, et al: Circulating biomarkers for detection of ovarian cancer and predicting cancer outcomes. Br J Cancer 110: 976-983, 2014.

17. Suryawanshi S, Vlad AM, Lin HM, Mantia-Smaldone G Laskey R, Lee M, Lin Y, Donnellan N, Klein-Patel M, Lee T, et al: Plasma microRNAs as novel biomarkers for endometriosis and endometriosis-associated ovarian cancer. Clin Cancer Res 19: $1213-1224,2013$.
18. Zheng H, Zhang L, Zhao Y, Yang D, Song F, Wen Y, Hao Q, Hu Z, Zhang $\mathrm{W}$ and Chen K: Plasma miRNAs as diagnostic and prognostic biomarkers for ovarian cancer. PLoS One 8: e77853, 2013.

19. Kan CW, Hahn MA, Gard GB, Maidens J, Huh JY, Marsh DJ and Howell VM: Elevated levels of circulating microRNA-200 family members correlate with serous epithelial ovarian cancer. BMC Cancer 12: 627, 2012.

20. Giordano S and Columbano A: MicroRNAs: New tools for diagnosis, prognosis, and therapy in hepatocellular carcinoma? Hepatology 57: 840-847, 2013.

21. Song B, Ji W, Guo S, Liu A, Jing W, Shao C, Li G and Jin G: miR-545 inhibited pancreatic ductal adenocarcinoma growth by targeting RIG-I. FEBS Lett 588: 4375-4381, 2014.

22. Du B, Wang Z, Zhang X, Feng S, Wang G, He J and Zhang B: MicroRNA-545 suppresses cell proliferation by targeting cyclin D1 and CDK4 in lung cancer cells. PLoS One 9: e88022, 2014.

23. Yiwei T, Hua H, Hui G, Mao M and Xiang L: HOTAIR interacting with MAPK1 regulates ovarian cancer skov3 cell proliferation, migration, and invasion. Med Sci Monit 21: 1856-1863, 2015.

24. Livak KJ and Schmittgen TD: Analysis of relative gene expression data using real-time quantitative PCR and the 2(-Delta Delta C(T)) method. Methods 25: 402-408, 2001.

25. Song B, Zhang C, Li G, Jin G and Liu C: MiR-940 inhibited pancreatic ductal adenocarcinoma growth by targeting MyD88. Cell Physiol Biochem 35: 1167-1177, 2015.

26. Zhou Q and Yu Y: Upregulated CDK16 expression in serous epithelial ovarian cancer cells. Med Sci Monit 21: 3409-3414, 2015.

27. Li H, Xu Y, Qiu W, Zhao D and Zhang Y: Tissue miR-193b as a novel biomarker for patients with ovarian cancer. Med Sci Monit 21: 3929-3934, 2015.

28. Agarwal V, Bell GW, Nam JW and Bartel DP: Predicting effective microRNA target sites in mammalian mRNAs. Elife: Aug 12: 4, 2015.

29. Cristiano BE, Chan JC, Hannan KM, Lundie NA, Marmy-Conus NJ, Campbell IG, Phillips WA, Robbie M, Hannan RD and Pearson RB: A specific role for AKT3 in the genesis of ovarian cancer through modulation of G2-M phase transition. Cancer Research 66: 11718-11725, 2006.

30. Zhang L, Volinia S, Bonome T, Calin GA, Greshock J, Yang N, Liu CG, Giannakakis A, Alexiou P, Hasegawa K, et al: Genomic and epigenetic alterations deregulate microRNA expression in human epithelial ovarian cancer. Proc Natl Acad Sci USA 105: 7004-7009, 2008.

31. Iorio MV, Visone R, Di Leva G, Donati V, Petrocca F, Casalini P, Taccioli C, Volinia S, Liu CG, Alder H, et al: MicroRNA signatures in human ovarian cancer. Cancer Res 67: 8699-8707, 2007.

32. Yang H, Kong W, He L, Zhao JJ, O'Donnell JD, Wang J, Wenham RM, Coppola D, Kruk PA, Nicosia SV and Cheng JQ: MicroRNA expression profiling in human ovarian cancer: miR-214 induces cell survival and cisplatin resistance by targeting PTEN. Cancer Res 68: 425-433, 2008.

33. Li N, Zhao X, Wang L, Zhang S, Cui M and He J: miR-494 suppresses tumor growth of epithelial ovarian carcinoma by targeting IGF1R. Tumor Biol 37: 7767-7776, 2016.

34. Yuan J, Wang K and Xi M: MiR-494 inhibits epithelial ovarian cancer growth by targeting c-Myc. Med Sci Monit 22: 617-624, 2016.

35. Song B, Ji W, Guo S, Liu A, Jing W, Shao C, Li G and Jin G: miR-545 inhibited pancreatic ductal adenocarcinoma growth by targeting RIG-I. FEBS Lett 588: 4375-4381, 2014.

36. Liao C, Xiao W, Zhu N, Liu Z, Yang J, Wang Y and Hong M: MicroR-545 enhanced radiosensitivity via suppressing Ku70 expression in Lewis lung carcinoma xenograft model. Cancer Cell Int 15: 56, 2015

37. Vivanco I and Sawyers CL: The phosphatidylinositol 3-kinase AKT pathway in human cancer. Nat Rev Cancer 2: 489-501, 2002.

38. Chin YR, Yoshida T, Marusyk A, Beck AH, Polyak K and Toker A: Targeting Akt3 signaling in triple-negative breast cancer. Cancer Res 74: 964-973, 2014.

39. Lin HP, Lin CY, Huo C, Jan YJ, Tseng JC, Jiang SS, Kuo YY, Chen SC, Wang CT, Chan TM, et al: AKT3 promotes prostate cancer proliferation cells through regulation of Akt, B-Raf, and TSC1/TSC2. Oncotarget 6: 27097-27112, 2015.

This work is licensed under a Creative Commons Attribution 4.0 International (CC BY-NC 4.0) License 